\title{
Interaction sol-fondation superficielle au voisinage de la crête d'un talus : analyse de la capacité portante
}

S. MALOUM

IUT Robert-Schuman de Strasbourg

72. route du Rhin

67400 IIlkirch-Graffenstaden

MaloumSihem@

netscape.net

\section{J.-G. SIEFFERT}

Institut de mécanique des

fluides et des solides

UMR 7507

École nationale des arts et industries de Strasbourg 24, bd de la Victoire

67084 Strasbourg Cedex jean-georges@ ensais.u-strasbg.fr
Parmi les problèmes géotechniques traités depuis des décennies, figure celui de l'interaction sol-fondation superficielle, toutes configurations confondues.

Le présent article traite du cas particulier du système solfondation superficielle filante établi au voisinage de la crète d'un talus, et sollicité par des chargements quasi statiques externes verticaux et inclinés centrés. La capacité portante est représentée par l'enveloppe des combinaisons de charges à la ruine, déterminées par simulations numériques aux éléments finis grâce au code CESAR-LCPC. Une comparaison effectuée avec les formulations issues du règlement et de la littérature sera présentée.

Mots-clés : fondation superficielle, talus, interaction, capacité portante, facteur réducteur, simulation numérique, éléments finis.

\section{Soil-shallow foundation interaction near the crest of a slope: bearing capacity analysis}

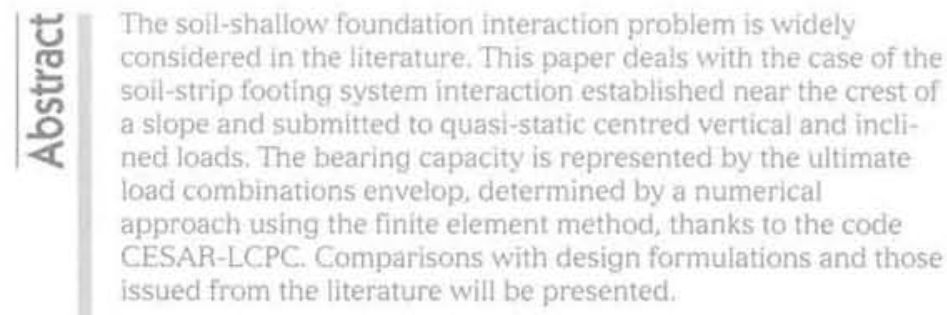

Key words : shallow foundation, slope, interaction, bearing capacity, reduction factor, numerical simulation, finite elements. 


\section{Introduction}

Du point de vue du génie civil, une structure n'est jamais isolée. Elle est le plus souvent en interactions multiples avec le sol d'assise, ainsi qu'avec les constructions avoisinantes.

Le degré d'importance des répercussions réciproques des comportements d'ouvrages appartenant à des domaines connexes est étroitement lié à leurs caractéristiques mécaniques et géométriques. Les réflexions sur les aléas, provenant du milieu extérieur, susceptibles d'affecter l'aptitude d'une structure à remplir ses fonctions par rapport à l'exploitation, à la sécurité et à l'environnement, ont amené les concepteurs à intégrer attentivement cette notion d'interaction lors de la phase de dimensionnement.

Dans cet article, on aborde le problème particulier de l'interaction sol frottant-fondation superficielle filante non encastrée de largeur B, établie à une distance d de la crête d'un talus. Ce dernier est caractérisé par son angle $\beta$ inférieur à l'angle de frottement interne du sol (Tableau I), ainsi que sa hauteur H (Fig. 1). Il est évident qu'une telle géométrie n'est pas sans conséquence directe sur la stabilité du système sol-fondation, quel que soit le chargement extérieur appliqué.

A noter que dans le cadre de cet article, on se limitera aux seuls cas de chargements statiques verticaux et inclinés centrés.

La présente étude a été réalisée dans l'optique de fournir une expression analytique de la capacité portante, représentée géométriquement dans un plan de sollicitations admissibles sous forme d'une enveloppe de combinaisons des charges à la ruine, autrement dit, d'enveloppe de rupture ou de surface de charge ultime (au sens de la théorie de la plasticité).

En raison de l'investissement financier important associé à l'investigation expérimentale, qu'il s'agisse d'essais de laboratoire ou d'essais en vraie grandeur, l'analyse du problème a été conduite par la méthode aux éléments finis. Le grand essor des approches numériques dans le domaine de la mécanique des structures et des géomatériaux permet en effet d'effectuer des calculs tenant compte de manière satisfaisante, des différentes non-linéarités géométriques et maté-

TABLEAU1 Paramètres retenus pour la stimulation. Constant parameters of the computations,

\begin{tabular}{l|c|c|c}
\hline \multicolumn{1}{c|}{ Paramétre } & Fondation & Interface & Sol \\
\hline $\mathrm{E}(\mathrm{kPa})$ & $71.10^{\circ}$ & $65.10^{3}$ & $65.10^{\circ}$ \\
\hline$v$ & 0,34 & 0,33 & 0,33 \\
\hline$\gamma\left(\mathrm{kN} / \mathrm{m}^{\top}\right)$ & & 16 & 16 \\
\hline$\varphi$ (angle de frottement interne) & & $37,5^{\circ}$ & $37,5^{\circ}$ \\
\hline $\mathrm{c}$ (cohésion, $\mathrm{kPa})$ & & 0,5 & 0,5 \\
\hline$\beta$ (inclinaison du talus) & & & $26,6^{\circ}$ \\
\hline$\psi$ (angle de dilatance du sol) & & $10^{\circ}$ & $10^{\circ}$ \\
\hline $\mathrm{R}_{1}$ (rèsistance à la traction, $\left.\mathrm{kPa}\right)$ & & 0,5 & \\
\hline
\end{tabular}

rielles, ainsi que d'effectuer des études paramétriques explorant diverses situations susceptibles d'être rencontrées dans la pratique.

\section{2}

\section{État de l'art}

A l'issue d'une synthèse bibliographique effectuée par Bakir (1993), et mise à jour par Maloum (2001), nous avons relevé l'intérêt majeur qu'a suscité, auprès de la communauté géotechnicienne, le thème de l'interaction sol-fondation superficielle de façon générale. Néanmoins, peu d'attention a été accordée à la configuration particulière du talus, en comparaison avec celle de la fondation sur plan horizontal.

A ce sujet, nous distinguons, parmi les travaux les plus significatifs, les études théoriques de de Buhan et Garnier (1994) et plus récemment de Soriano et al. (2001), fondées sur l'approche du calcul à la rupture, ainsi que les simulations physiques réalisées à la centrifugeuse du Laboratoire central des ponts et chaussées (LCPC) de Nantes par Bakir (1993) et Maréchal (1999). Ces derniers ont exprimé mathématiquement les coefficients réducteurs de la portance, en fonction du type de chargement appliqué au sol par l'intermédiaire de la fondation.

Pour ce qui est du cas de la fondation filante sollicitée par un chargement vertical centré, Bakir (1993) a proposé d'écrire le facteur $i_{\beta}$ sous la forme:

$$
i_{\beta}=1-0,9 \tan \beta(2-\tan \beta)\left\{1-\frac{d}{6 B}\right\}^{2}
$$

où l'on voit apparaitre l'angle $\beta$ du talus ainsi que le paramètre adimensionnel $\frac{d}{B}$, exprimant la distance
relative fondation-talus.

Cette formulation a été adoptée par le Rẻglement français fascicule 62-titre V (1993), après une modification effectuée au niveau du dernier terme, dans lequel le rapport $\frac{d}{6 B}$ a été substitué par $\frac{d}{8 B}$, induisant une majoration de la capacité portante de la fondation superficielle au voisinage d'un talus.

En ce qui concerne les chargements inclinés centrés, la « non-symétrie » de la surface libre du sol impose de traiter distinctement les cas des inclinaisons positives et négatives. Les figures $2 \mathrm{a}$ et $2 \mathrm{~b}$ schématisent la convention de signes que nous avons. Celle-ci indique que la charge est inclinée positivement $(\delta>0)$ par rapport à l'axe vertical de la fondation, lorsqu'elle est dirigée vers le talus.

Dans ce demier cas, le fascicule 62 propose d'exprimer le facteur $i_{\overline{ }}$ suivant l'équation (2). Ce facteur traduit la réduction de la capacité portante du système sol-fondation, générée par les effets couplés de l'inclinaison $\delta$ du chargement et de l'angle $\beta$ du talus.

$$
i_{\delta \beta}=\left(1-\frac{\delta+\beta}{45}\right)^{2}
$$

$\beta^{\prime}$ correspond à une inclinaison donnée de talus que l'on détermine en supposant l'égalité entre les facteurs réducteurs $i_{\delta}$ pour une inclinaison $\delta=\beta^{\prime}$, et $i_{\beta}(1)$.

Il convient ici de préciser que $i_{g}$ rend compte de la réduction de la capacité portante d'une fondation 


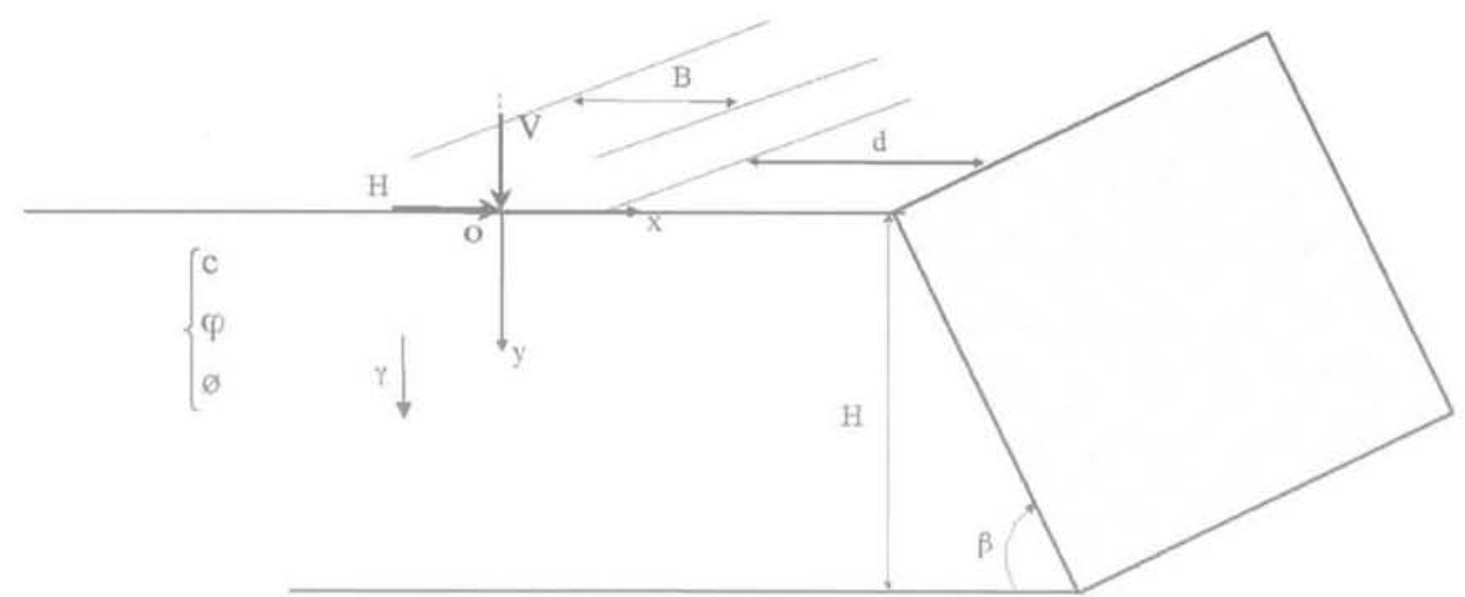

FIG: 1 Géométrie du problème.

Problem geometry.

superficielle établie sur un plan horizontal, et soumise à un chargement incliné centré. A ce titre, le fascicule 62 recommande, pour une fondation simplement posée sur un sol pulvérulent, l'utilisation de l'expression (3) :

$$
i_{5}=\left(1-\frac{|\delta|}{45}\right)^{2}
$$

Il suit alors immédiatement :

$$
\beta^{\prime}=45\left(1-\sqrt{i_{\beta}}\right)
$$

Ce principe d'égalité est fondé sur une hypothèse de similarité entre deux configurations de géométrie et de chargement complètement différentes : d'une part, une fondation établie sur un plan horizontal, et sollicitée par un chargement centré incliné de $\beta^{\prime}$ par rapport à son axe vertical et, d'autre part, une fondation située au voisinage d'un talus d'angle $\beta^{\prime}$, et sollicitée par un chargement vertical centré.

Au sens des auteurs, ce raisonnement ne repose sur aucun fondement physique clair. A ce propos, une méthode plus significative de détermination de l'angle $\beta$ ' sera proposée ultérieurement.

Il convient en outre de noter que Maréchal (1999) a montré, en s'appuyant sur l'analyse de ses résultats issus d'essais centrifugés, que pour les chargements inclinés positivement, l'expression (2) tend à sous-esti- mer les résultats expérimentaux. Il préconise de la substituer par l'équation (5), jugée plus conservative.

$$
i_{\delta \beta}=\left|i_{5}\right| i_{\beta}
$$

Pour ce qui est du cas de la charge extérieure orientée vers le plan horizontal, le fascicule 62 recommande d'adopter le facteur réducteur $i_{\delta \beta}$ ci-dessous :

$$
i_{\delta \beta}=\inf \left\{i_{s}\left(1-\frac{|| \delta|-\beta|}{45}\right)^{2}\right.
$$

L'égalité (6) permet une détermination par parties de $i_{\sigma \beta}$ dans le cas de chargements inclinés négatifs, en fonction de l'importance de l'inclinaison de la charge par rapport à l'axe vertical de la fondation.

\section{3}

\section{Simulation numérique par éléments finis}

Le logiciel aux éléments finis CESAR-LCPC a été choisi pour constituer une banque de données sur les charges de ruine et les déplacements induits du système sol-fondation filante au voisinage d'un talus, sollicité par des chargements verticaux et inclinés centrés.

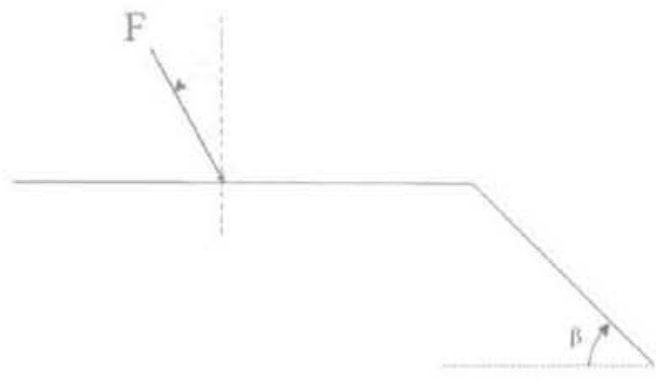

FG. 2a Inclinaison positive de la charge. Positive load inclination.

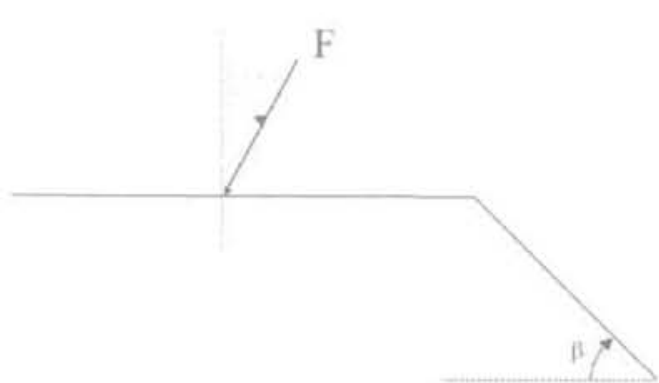

H6. 26 Inclinaison négative de la charge. Negative load inclination. 
Tous les calculs ont été effectués en considérant un état de déformation plane, étant donné le type de fondation retenu.

\section{1}

\section{Discrétisation spatiale du système sol-fondation}

Le massif de sol ne présentant aucune symétrie géométrique, il a donc été discrétisé dans son intégralité, par des éléments finis de type quadratique isoparamétrique à 8 nceuds. Le même type d'éléments a été adopté pour le maillage de la fondation, afin d'assurer un assemblage correct.

Un raffinement local du maillage a été effectué dans les zones suspectées à forts gradients de contraintes : au voisinage de la fondation, sous sa base, ainsi qu'au niveau du talus.

De manière générale, les dimensions du maillage ont été dẻfinies de façon à éviter toute source de perturbation à la formation des mécanismes de ruine dans le sol.

Les conditions aux limites sur toutes les frontières du massif, ainsi qu'un exemple de maillage sont illustrés par la figure suivante (Fig. 3).

\section{2}

\section{Caractéristiques de l'analyse numérique}

Le comportement du sol est régi par une loi élastique parfaitement plastique et le critère de frottement adopté correspond à celui de Mohr-Coulomb. Les valeurs des paramètres du calcul $(E, v, \gamma$ et $\varphi$ ) mentionnées sur le tableau I ont été déterminées expérimentalement par Maréchal (1999). Elles correspondent au sable de Fontainebleau utilisé lors de ses essais au LCPC de Nantes.

Pour ce qui est de la faible cohésion affectée au sol frottant, bien qu'il soit supposé parfaitement sec, son rôle est de réduire certaines instabilités numériques notées lors des simulations numériques Maloum (2001). L'angle de dilatance $\psi$ est choisi inférieur à l'angle de frottement interne $\varphi$, pour tenir compte du caractère non associatif de l'écoulement plastique.

Le matériau de la fondation filante est supposé, quant à lui, suivre un comportement de type élastique linéaire isotrope. Sa rigidité importante par rapport à celle du sol permet d'assimiler la cinématique de la fondation à celle d'un corps rigide.

En ce qui concerne les éventuels mécanismes de type décollement/glissement localisés à l'interface solfondation, ils ont été pris en compte par l'introciuction d'éléments de contact spécifiques sans épaisseur à 6 nceuds, vérifiant les critères de frottement et d'adhérence. Les caractéristiques élastiques ainsi que le frottement d'interface sont supposés ètre identiques à ceux du sol d'assise. Le critère d'adhérence, quant à Iui, a été caractérisé par une faible résistance à la traction (R.), le but étant, de la même manière que pour la cohésion, de remédier à certaines instabilités numériques.

Une étude paramétrique a ciblé deux paramètres : un premier de nature géométrique correspondant à la distance relative au talus $\frac{d}{B}$, et un second lié au chargement caractérisé par le signe et la valeur de l'inclinaison $\delta$.

\section{3}

\section{Normalisation adoptée}

Dans l'objectif d'affranchir la discussion du facteur de capacité portante $N_{y}$ dont la détermination a suscité tant de controverses Sleffert et Bay-Gress (1999), Paolucci et Pecker (1997) ont proposé de normaliser les composantes horizontales et verticales du chargement extérieur $(\mathrm{H}, \mathrm{V})$ par la charge ultime notée $\mathrm{V}_{\text {mix }}$, relative à la configuration de référence. Celle-ci correspond au cas de la fondation superficielle filante établie sur un massif à surface libre horizontale, et soumise à un char-

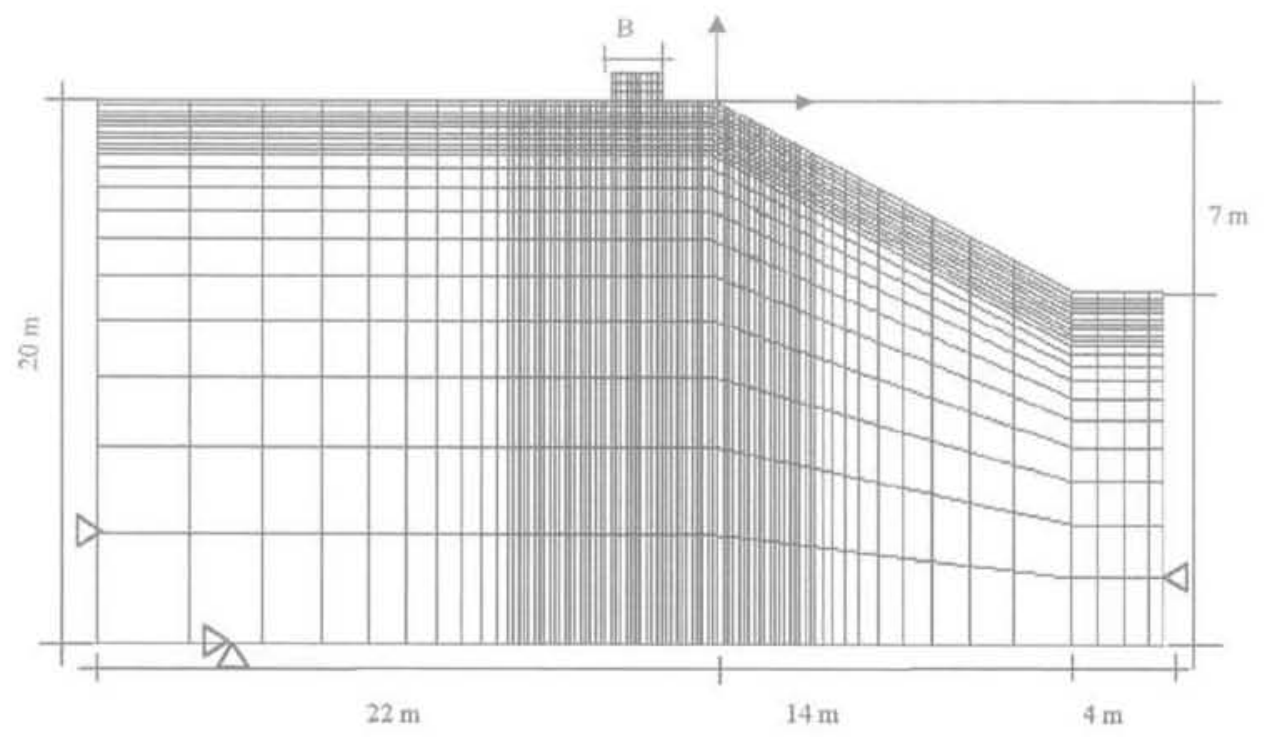

FG. 3 Maillage du modèle bidimensionnel et conditions aux límites. Finite element mesh of the model and boundary conditions. 


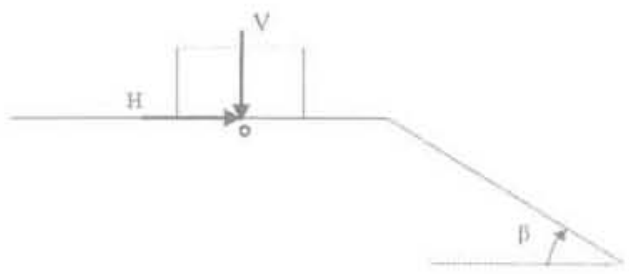

FIG, 4a Torseur des efforts extérieurs. External forces.

gement vertical centré. Les composantes de chargement normées obtenues sont représentées sur la figure 4a et données par:

$$
\left\{\begin{array}{l}
y \\
H
\end{array}\right\}=\frac{1}{V_{\max }}\left\{\begin{array}{l}
Y \\
H
\end{array}\right\}
$$

Quant aux composantes cinématiques, autrement dit le tassement S et le déplacement horizontal U, elles ont été rendues adimensionnelles en les divisant simplement par la largeur B de la fondation.

$$
\left\{\begin{array}{l}
s \\
u
\end{array}\right\}=\frac{1}{B}\left\{\begin{array}{l}
s \\
v
\end{array}\right\}
$$

A noter que le point de réduction des composantes statiques et cinématiques est ramené au centre de la base de la fondation, matérialisé par le point $\mathrm{O}$, en contact avec le sol.

\section{4}

\section{Analyse des résultats}

Avant de procéder à l'analyse des résultats obtenus, il nous semble intéressant d'évoquer deux problématiques rencontrées lors des calculs numériques :

(i) Il s'agit, dans un premier temps, du mode d'application du chargement à la fondation. Quel que soit le chemin de chargement extérieur, son application au nœud central de la base de la fondation s'est effectuée

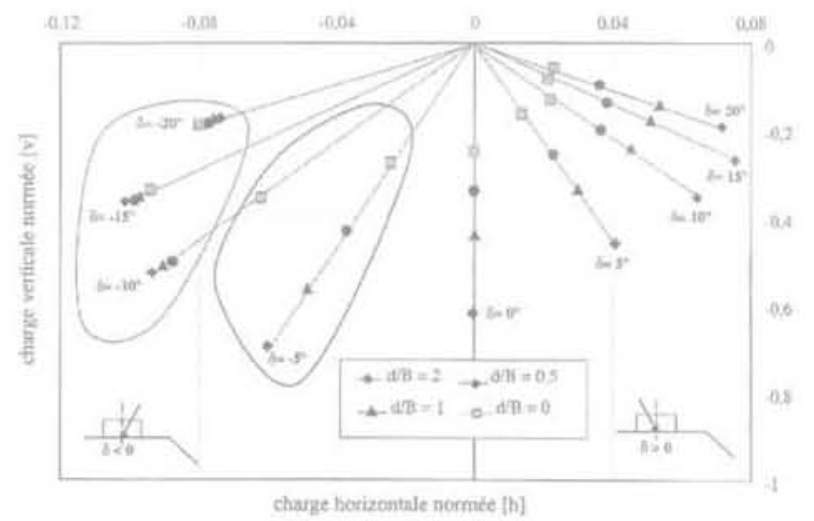

FG.5 Influence de la distance relative fondationtalus sur la capacité portante.

Influence of the relative distance footing-slope on the bearing capacity.

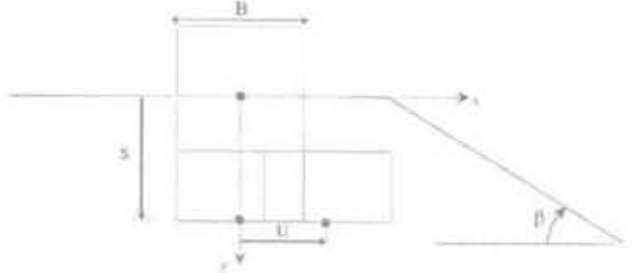

FiG. 46 Cinématique de la fondation. Foundation kinematics.

selon un mode incrémental irrégulier. En effet, on recommande généralement à l'initiation du chargement ainsi qu'aux alentours de la ruine, l'introduction de faibles incréments de charge, contribuant ainsi à une convergence plus aisée du processus itératif.

(ii) Quant au second point, il est relatif à la question de la détermination de la charge limite, qui demeure encore aujourd'hui très ambiguë aussi bien pour le numéricien que pour l'expérimentateur. Dans le cas de nos simulations numériques, il a été supposé que celleci correspond à l'initiation des grands déplacements de la fondation, matérialisée physiquement par un changement brutal de la direction de la réponse chargedéplacement.

La figure 5 illustre les charges ultimes correspondant aux chemins de chargement testées, dans le plan normé (h, v) des composantes des sollicitations admis-

sibles. L'influence du paramètre adimensionnel $\frac{d}{B}$ est de toute évidence de premier ordre quant à l'étude de la stabilité du système interactif sol-fondation superficielle au voisinage d'un talus. On observe au fur et à mesure que la fondation s'approche de la crête du talus, une forte réduction de sa capacité portante, notamment lorsque l'inclinaison $\delta$ est positive ou nulle.

En revanche, pour ce qui est des chargements négatifs, le talus ne semble pas influencer les fortes inclinaisons (considérées en valeurs absolues). Ce constat n'est cependant plus valable lorsqu'il devient question des charges négatives faibles, Dans cette situation, on note des écarts importants entre les valeurs des charges de ruine, en fonction de la position de la fondation par rapport au talus.

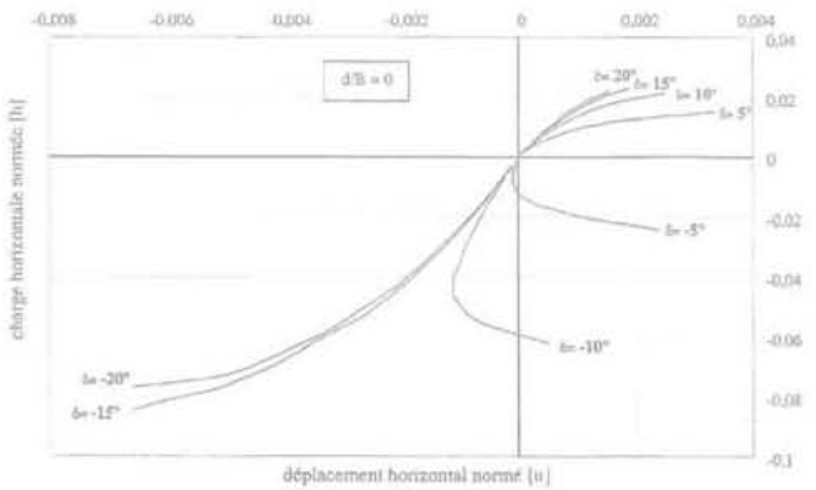

FG.6 Comportement dans la direction horizontale sous chargements centrés inclinés $(\mathrm{d} / \mathrm{B}=0)$.

Horizontal behavjour under centred inclined load paths $(\mathrm{d} / \mathrm{B}=0)$ 
TABLEAUII Paramètres variables de la simulation. Variable parameters of the computations.

\section{Paramètres}

\begin{tabular}{l|l} 
Symbole & Valeurs \\
\hline$\frac{d}{B} \begin{array}{c}\text { distance relative } \\
\text { fondation-talus) }\end{array}$ & $0 ; 0,5 ; 1 ; 2 ;+\infty$ \\
\hline$\delta$ (inclinaison de la charge) & $\pm 5^{\circ} ; \pm 10^{\circ} ; \pm 15^{\circ} ; \pm 20$ \\
\hline
\end{tabular}

Pour tenter de comprendre les raisons de cette variation, on propose d'observer et d'analyser les réponses dans la direction horizontale (h-u) illustrées par la figure 6, pour la configuration géométrique la plus défavorable $\frac{d}{B}=0$.

Les réponses relatives à $\delta=-5^{\circ}$ et $-10^{\circ}$, attestent d'un comportement particulièrement intéressant : au début du chargement, la courbe semble suivre normalement la direction du chargement négatif, jusqu'à atteindre un certain niveau de sollicitation, à partir duquel les déplacements horizontaux affichent un changement de signe rapide et définitif.

Cette évolution, quelque peu surprenante, a conduit à s'interroger sur les raisons associées aux déviations soudaines des directions de ces deux trajectoires de déplacement. Il s'avère que le changement de signe des déplacements horizontaux correspond à l'initiation d'un mécanisme de ruine qui intercepte plutôt le talus et non pas le plan horizontal, comme s'il s'agissait d'un chargement incliné positivement (vers le talus). Ce phénomène est primordial quant au suivi d'un ouvrage. Il convient d'en tenir compte dans les phases de la construction.

Pour analyser plus finement le problème, on propose d'examiner les trajectoires de déplacement relatives à l'inclinaison de charge la plus faible $\delta=-5^{\circ}$ en fonction de $\frac{d}{B}$ (Fig. 7). Celles-ci mettent en évidence un comportement qu'on peut décrire d'évolutif, fortement lié à la position de la semelle par rapport à la crête du talus. Le mécanisme de ruine change de forme pour une valeur de $\frac{d}{B}$ comprise entre 1 et 2 (en l'occur-

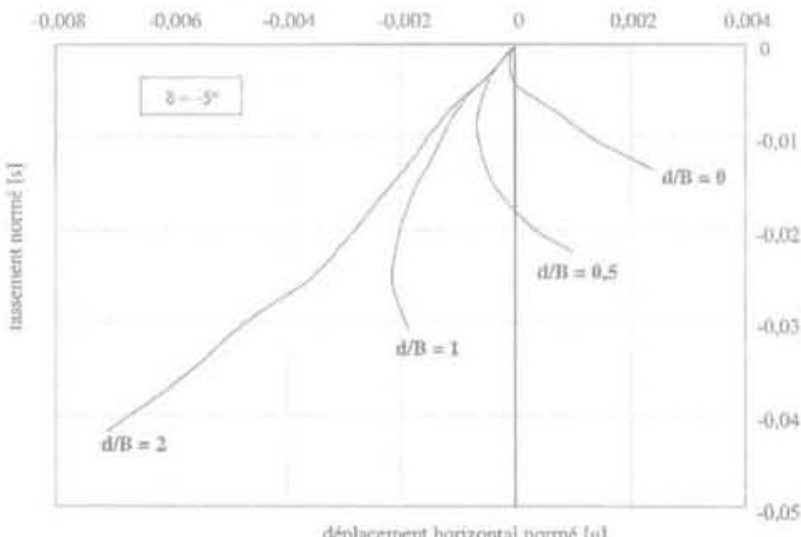

FIG.7

Évolution de la direction de la trajectoire de déplacement en fonction de $\mathrm{d} / \mathrm{B}\left(\delta=-5^{\circ}\right)$. Evolution of the displacement trajectory direction according to $\mathrm{d} / \mathrm{B}\left(\delta=-5^{\circ}\right)$. rence ici pour le cas $\delta=-5^{\circ}$ ) que nous qualifions de « distance relative de transition $)$.

En ce qui concerne les cas $\frac{d}{B}=0 ; 0,5$ et 1 , la ruine se produit par formation d'un mécanisme qui intercepte le talus, alors que pour $\frac{d}{B}=2$, la ruine se développe normalement dans la direction du plan horizontal.

\section{Expression analytique de l'enveloppe de rupture}

A l'issue de cette analyse, il nous a èté possible de conclure de l'existence d'un angle qu'on note $\delta$, ('indice s fait référence à simulation numérique), quì correspond à l'inclinaison de la charge extérieure marquant la transition entre les deux mécanismes de rupture, évoqués précédemment.

Les résultats des charges ultimes, obtenues par approche numérique, sont représentés sur le plan normé (h, v), qui peut être scindé en une zone influencée par le talus et une autre non influencée. L'écriture de la surface de charge ultime s'effectue donc par parties : à gauche de la droite formant l'angle $\delta_{\text {r }}$ avec l'axe des composantes de sollicitations verticales (Fig. 8), on retient la formulation proposée par Le Pape (2000), dans le cas d'une fondation superficielle filante sur plan horizontal.

$$
f_{\text {ult }(h, v)}=h+c_{2} \cdot v \cdot \ln \left(\frac{1}{v}\right)=0
$$

Pour tenir compte des effets générés par le talus, on adopte pour la partie de droite une expression simjlaire, dans laquelle a été introduit le facteur réducteur de la portance sous charge verticale centrée.

$$
f_{u n t}(h, v)=h-c_{-1} \cdot v \cdot \ln \left(\frac{i_{\beta}}{v}\right)=0
$$

Les coefficients $c$, et $c_{2}$ décrivent la forme de l'enveloppe de rupture totale.

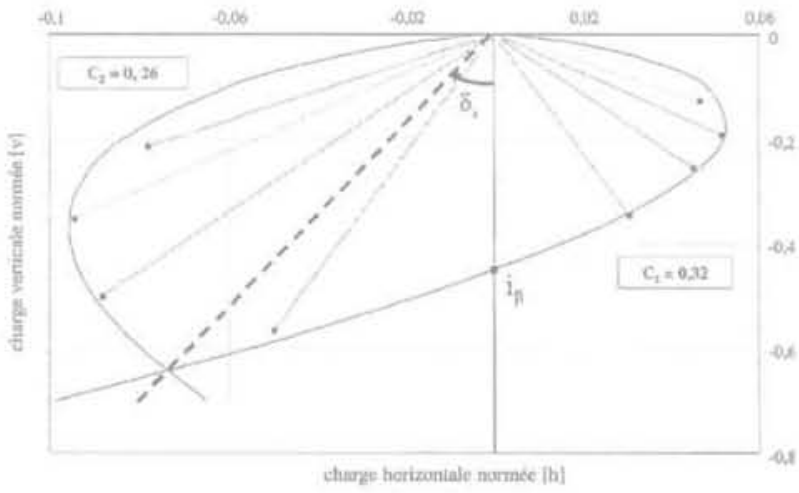

FG.8 Enveloppe de rupture totale $\left(\frac{d}{B}=1\right)$. Complete failure envelope $\left(\frac{d}{B}=1\right.$. 


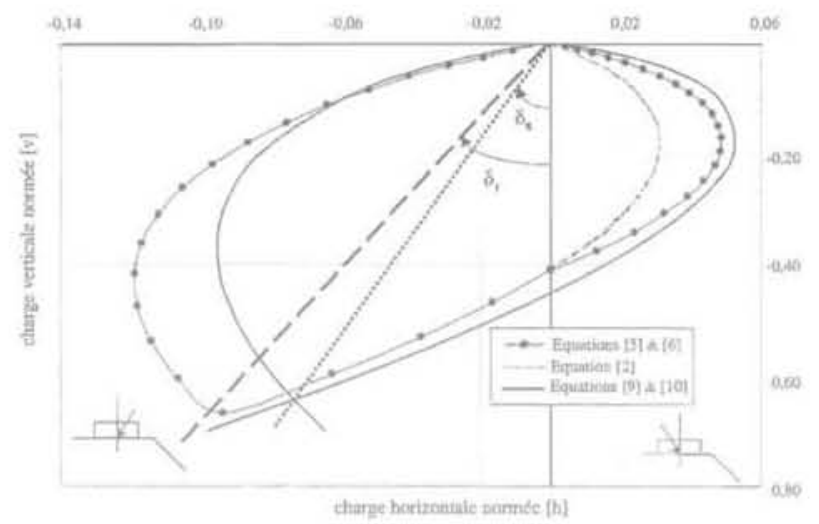

FIG.9 Enveloppes de rupture selon diverses approches.

Rupture envelopes following different approaches.

\section{6}

\section{Étude comparative}

Les enveloppes de rupture issues des formulations du fascicule 62-titre $V(2)$ et (6) des travaux de Maréchal (1999) (5) et, enfin, de nos simulations numériques (9) et (10) sont illustrées par la figure 9. Deux remarques sont à noter :

1) A droite de la ligne, en traits interrompus, formant un angle $\delta_{\text {f }}$ (de la mème manière que précédemment, l'indice f ici fait référence au fascicule 62-titre V) avec l'axe des sollicitations verticales, on constate que nos résultats numériques sont assez proches de ceux obtenus par application du règlement, et ce quel que soit le signe de l'inclinaison du chargement imposé. Cependant, la divergence est notable en ce qui concerne la région située à gauche de cette ligne, oủ le mécanisme de ruine n'est pas influencé par le talus. Les charges limites correspondant à la formulation du fascicule 62 -titre V (6) sont nettement supérieures à celles obtenues numériquement;

2) Les valeurs des inclinaisons de transition $\delta_{\text {s }}$ et $\delta$ marquant le changement du mécanisme de ruine respectivement dans les cas de nos simulations numériques et du fascicule 62, sont nettement différentes.

Avant de conclure ce travail, on propose d'évaluer quantitativement l'angle $\beta^{\prime}$ (4), comme il a été précisé précédemment, en l'exprimant en fonction de l'inclinaison de transition $\delta$. Étant donné que l'équation (6) stipule que le facteur réducteur $i_{s g}$ relatif aux chargements inclinés négativement, s'exprime en deux parties. Par conséquent, on peut écrire lorsque l'inclinaison du chargement extérieur $\delta$ est égale à $\delta$ :

$$
i_{\delta_{i, \beta}}=\left(1-\frac{\left(\left|\delta_{f}\right|-B^{\prime}\right)}{45}\right)^{2}=\left(1-\frac{\left|\delta_{f}\right|}{45}\right)^{2}
$$

Il en découle alors :

$$
\left|\delta_{\gamma}\right|=\frac{B^{\prime}}{2}=22,5\left(1-\sqrt{i_{\beta}}\right)
$$

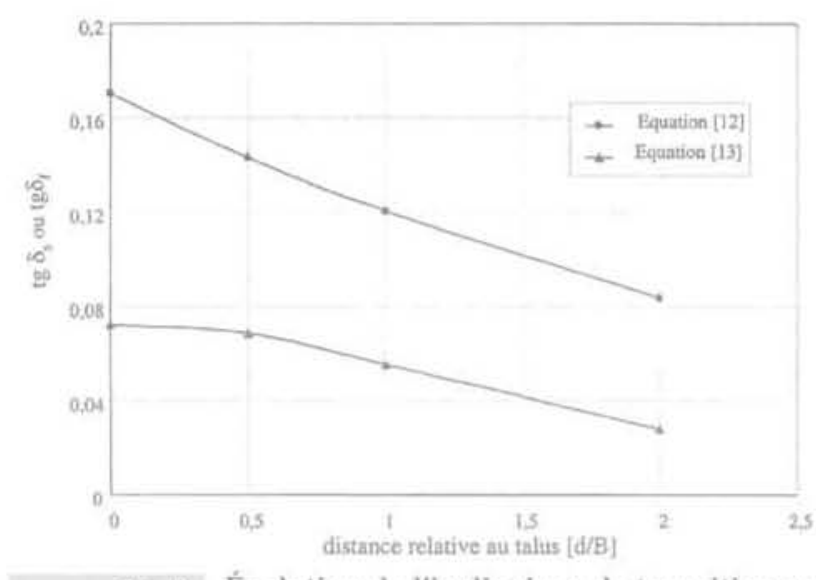

FG.10 Évolution de l'inclinaison de transition en fonction de $\frac{d}{B}$.

Evolution of the transition inclination according to $\frac{d}{B}$.

A présent, afin de mieux visualiser les écarts, évoqués dans la remarque (2), entre les inclinaisons marquant la transition entre les mécanismes de rupture prédéfinis, obtenues selon les formulations du fascicule 62 -titre V (12) et nos résultats numériques (13) la figure 10 représente graphiquement les évolutions de $\tan \delta_{\mathrm{s}}$ et de $\tan \delta_{f}$, en fonction de la distance relative fondationtalus.

A noter que l'expression (13) ci-dessous est déduite de l'égalisation des équations (9) et (10) relatives aux enveloppes de rupture numériques.

$$
\tan \delta_{\mathrm{s}}=\frac{c_{1} c_{2}}{c_{1}+c_{2}} \ln \left(i_{\beta}\right)
$$

\section{7}

\section{Conclusion}

Le travail présenté a porté sur la capacité portante du système sol frottant sec-fondation superficielle filante au voisinage d'un talus, en considérant l'effet des interactions multiples générées par le couplage de plusieurs facteurs liés à la géométrie de la fondation ( $B$, d), à celle du sol d'assise $(\beta, H)$, ainsi qu'à l'inclinaison $\delta$ du chargement appliqué au centre de la base de la semelle,

L'apport principal de l'étude se situe dans l'expression mathématique de l'enveloppe des combinaisons des charges à la ruine, relatives au système interactif sol-fondation, en fonction du chargement qui lui est appliqué.

L'incidence de la distance relative fondation-talus $\frac{d}{B}$ sur la réduction de la portance a été clairement mise en évidence.

Enfin, la variation du mécanisme de ruine en fonction du chargement extérieur appliqué est un paramètre déterminant quant au suivi d'un ouvrage établi au voisinage de la crête d'un talus. 